\title{
Negativer Transfer beim Gebrauch des Definitartikels von chinesischen Lernenden des Deutschen als L2: Fehleranalyse
}

\section{Negative transfer in the use of the definite article by Chinese L2-learners of German: an error analysis}

\author{
Gisella FERRARESI' 10
}

${ }^{1}$ Prof. Dr., Marmara Üniversitesi, Fen Edebiyat Fakültesi, Alman Dili ve Edebiyatı Anabilim Dalı, İstanbul, Türkiye

ORCID: G.F. 0000-0001-5349-3214

\section{Corresponding author:}

Gisella FERRARESI,

Marmara Üniversitesi, Göztepe Kampüsü,

Fen Edebiyat Fakültesi, Alman Dili ve

Edebiyatı Anabilim Dalı, 34722, Kadıköy,

İstanbul, Türkiye

E-mail: ferraresigisella@gmail.com

Submitted: 10.11 .2019

Revision Requested: 27.11.2019

Last Revision Received: 04.12.2019

Accepted: 13.12 .2019

Citation: Ferraresi, G. (2019). Negativer Transfer beim Gebrauch des Definitartikels von chinesischen Lernenden des Deutschen als L2: Fehleranalyse. Alman Dili ve Edebiyatı Dergisi - Studien zur deutschen Sprache und Literatur, 42, 19-38.

https://doi.org/10.26650/sdsl2019-0018

\begin{abstract}
DEUTSCH)
In diesem Aufsatz wird eine Fehleranalyse zum Gebrauch des Definitartikels bei chinesischen Lernenden des Deutschen als L2 vorgenommen, um der Frage nachzugehen, wie die L1 Chinesisch als artikellose Sprache das Lernen des Definitartikels negativ beeinflusst. Nach Hawkins \& Filipović (2012) wirken sich verschiedene Faktoren negativ oder positiv auf das Lernen im L2-Erwerb aus, darunter auch die typologischen Unterschiede zwischen der L1 und der L2. Das Chinesische unterscheidet sich typologisch vom Deutschen u.a. auch dadurch, dass Definitheit im Deutschen morphosyntaktisch durch den Definitartikel realisiert wird, im Chinesischen hingegen die definite Interpretation der Nominalphrasen durch Strategien wie z. B. die Wortstellung bewirkt wird. Demzufolge stellt das Lernen eines Artikelsystems Sprecher einer artikellosen Sprache vor große Schwierigkeiten, die dadurch verschärft werden, dass bei der Interpretation eines Nomens als definit nicht nur semantische, sondern auch pragmatische Faktoren eine Rolle spielen, wie die verschiedene Gebrauchskontexte des Definitartikels von Himmelmann (1997) zeigen. In der empirischen Studie wurden von chinesischen Lernenden des Deutschen frei geschriebene Texte für Nominalphrasen untersucht. Die Texte waren Motivationsschreiben von 80 chinesischen Bewerberinnen für den Masterstudiengang Germanistik: Sprachwissenschaft an der Otto-FriedrichUniversität Bamberg in der Zeit vom Wintersemester 2014/15 bis zum Sommersemester 2018. Die erhobenen Nominalphrasen wurden zuerst qualitativ nach den semantisch-pragmatischen Kategorien von Himmelmann (1997) klassifiziert und dann in Hinblick auf die zielsprachliche Realisierung quantitativ analysiert.

Die Analyse der Daten zeigt, dass fortgeschrittene chinesische Lernende Nomen tendenziell als nackt realisieren, wenn diese zunächst schon erwähnt worden sind und deren Interpretation kontextuell rekonstruierbar ist. Ähnliches gilt für die Topikposition.
\end{abstract}

Schlüsselwörter: L2-Erwerb, Fehleranalyse, Definitartikel, artikellose Sprachen, pragmatische Faktoren 


\section{ABSTRACT (ENGLISH)}

In this essay, an error analysis of the use of the definitive article in Chinese learners of German as L2 is undertaken to investigate how L1 Chinese as an article-less language affects the acquisition of German in terms of definiteness.

According to Hawkins \& Filipović (2012), various factors have a negative or positive effect on L2 learning, including the typological differences and similarities between L1 and L2. Chinese is typologically different from German, where definiteness is morphosyntactically realized by the definite article, whereas in Chinese the definite interpretation of the noun phrases is brought about by strategies such as word order. Consequently, the learning of an article system poses great difficulties for speakers of an article-less language, which are further complicated by the fact that not only semantic factors but also pragmatic ones play a role in the interpretation of a noun, as the different usage contexts of the definite article by Himmelmann (1997) show.

In the empirical study, freely written texts by Chinese learners of German are examined for noun phrases. The texts were the motivational letters of 80 Chinese applicants for the master's program in German Studies: Linguistics at the Otto-FriedrichUniversity of Bamberg in the period from the winter term 2014/15 to the summer term 2018. The noun phrases were first analyzed qualitatively according to the semantic-pragmatic categories of Himmelmann (1997) and then quantitatively with regard to their target-language realization.

The analysis of the data shows that advanced Chinese learners tend to realize nouns as bare in case they have been mentioned before, and their interpretation can be contextually reconstructed. The same applies to the topic position.

Keywords: L2 acquisition, error analysis, definite article, article-less languages, pragmatic factors

\section{EXTENDED ABSTRACT}

In this paper, an error analysis of the use of the definitive article by Chinese learners of German as L2 is undertaken in order to investigate how the L1 Chinese as an articleless language influences the acquisition of German in terms of definiteness, following the model of the Complex Adaptive System Principle of Hawkins \& Filipovic (2012).

According to this multifactorial model for learning in L2 acquisition, several factors can have a positive or negative effect on learning in L2 acquisition, including the typological differences or similarities between L1 and L2. Chinese is typologically different from German a.o. in that definiteness in German is realized morphosyntactically by the definite article, whereas in Chinese no articles are realized. The definite interpretation of noun phrases is affected by other strategies such as word order. From a typological point of view, in most languages of the world - as in Chinese - there are no articles, or only the definite or indefinite articles are realized, as for example in Turkish. Languages in which both are realized, such as German or English, tend to be the exception. As a result, learning an article system poses great difficulties to speakers of an article-less language

Numerous studies address different aspects of article system acquisition by learners with an article-less L1. Some publications such as Breckle \& Zinsmeister (2010), for 
example, examine whether specificity or definiteness as semantic components play a more important role in the target-language realization of the article. However, none of the studies deals with the concrete typological differences between the L1 and the L2.

The notion of definiteness itself is a very complex concept that consists of different aspects, because not only semantic but also pragmatic factors play a role in the interpretation of a noun as definite, as Himmelmann (1997) discusses in detail. Himmelmann (1997) proposes various usage contexts for the use of the definitive article, which are also relevant for the empirical study.

In order to answer the initial question, a corpus was created with texts written by Chinese learners of German. The corpus consists of 80 letters of motivation from Chinese applicants for the master's program in German Studies: Linguistics at the OttoFriedrich-University of Bamberg during a period from the winter term 2014/15 to the summer term 2018. For the error analysis, all noun phrases with definite, indefinite and zero-article were categorized.

The noun phrases within the three groups were then differentiated into targetlanguage and non-target-language realizations. The definite noun phrases were first qualitatively classified according to the semantic-pragmatic categories of Himmelmann (1997) and then quantitatively analyzed with regard to their target-language realization.

The analysis of the data shows that even advanced Chinese learners tend to realize nouns as bare once they have been mentioned, and their interpretation can be contextually reconstructed. Fewer errors appear with those definite noun phrases that are realized in anaphoric-contextual contexts. In Chinese, the demonstrative pronoun is used in such contexts, so that the Chinese structure is very similar to the German one. In contrast, the abstract-situative context seems to be difficult for Chinese learners, because in this context noun phrases in Chinese are always realized bare. Among the semantic contexts there are often also nouns that have a generic interpretation.

Such typological considerations should also be taken into account in teaching this difficult grammatical area of the German language. 


\section{Einleitung'}

Das Lernen des Artikelsystems stellt Lernende mit einer artikellosen L1 vor erhebliche Schwierigkeiten. Filipović \& Hawkins $(2013)^{2}$ legen in ihrer Diskussion über die verschiedenen Faktoren, die das Lernen im L2-Erwerb positiv oder negativ beeinflussen können, ihre Aufmerksamkeit unter anderem auf die typologischen Unterschiede zwischen der L1 und der L2 ${ }^{3}$. Ihre Studie zum Erwerb des Artikelsystems durch L2Lernende, deren L1 artikellos ist, zeigt, dass die L1 den L2-Erwerb negativ beeinflusst. In diesem Beitrag gehe ich der Frage nach, inwiefern die L1 Chinesisch sich negativ auf den Erwerb des Definitartikels im Deutschen auswirkt. Ziel dieses Beitrags ist, anhand einer Fehleranalyse bzw. der Analyse der zielsprachlichen realisierten Nominalphrase aus einem selbst erstellen Korpus zu zeigen, dass manche Typen von definiten Nominalphrasen weniger häufig von Fehlern betroffen sind als andere, da Definitheit wenn auch nicht morphosyntaktisch - im Chinesischen doch auch realisiert wird. Für die Klassifikation der Typen von definiten Nominalphrasen im Deutschen wurden die Gebrauchskontexte von Himmelmann (1997) übernommen.

Das Korpus besteht aus 80 von chinesischen Studierenden verfassten Texten. Bei den Texten handelt es sich um Motivationsschreiben chinesischer Studierender, die sich in dem Zeitraum zwischen dem Wintersemester 2014/15 und dem Sommersemester 2018 für den Masterstudiengang 'Germanistik: Sprachwissenschaft' an der Otto-FriedrichUniversität Bamberg bewerben wollten.

Das Thema des negativen Transfers der L1 auf die L2 wird im folgenden Abschnitt 2 diskutiert, während die typologischen Unterschiede in der Realisierung der Definitheit im Chinesischen und im Deutschen im Abschnitt 3 dargestellt werden. In diesem Abschnitt wird auch auf den komplexen Begriff der Definitheit näher eingegangen, da auch in den chinesischen Grammatiken und Lehrwerken der Definitartikel als morphosyntaktischer

1 Ich danke beiden Gutachtern für wertvolle Kommentare.

2 Die beiden Gutachter merken an, dass die Begriffe 'Lernen' und 'Erwerb' unterschieden werden müssten. Beim 'Lernen einer Fremdsprache' wird meistens eine Sprache bewusst und gezielt im Unterrichtskontext erworben. Dabei spielen auch pädagogische Überlegungen eine Rolle. Der Erwerbsprozess hingegen beinhaltet eher Faktoren wie Alter, Einfluss der L1-Sprache, Verarbeitungsmechanismen, wie auch Filipović \& Hawkins (2013, S. 12) behaupten: "It has been noted many times by different scholars that second language acquisition involves the interplay of a number of factors that can either facilitate or impede learning." Die Autoren selbst definieren ihr Complex Adaptive System Principle (CASP) als "a set of principles for a multi-factor model of learning in second language acquisition." Ich benutze hier die Begriffe 'L2-Erwerb' und 'Lernen' nach Hawkins \& Filipović (2012) und Filipović \& Hawkins (2013).

3 Die typologischen Unterschiede wirken sich nicht immer nur negativ auf den L2-Erwerb aus. 
Ausdruck der Kategorie Definitheit gilt, der Begriff der Definitheit jedoch weitaus komplexer ist und aus mehreren Aspekten besteht. Neben der Realisierung des Definitartikels im Deutschen nach den Gebrauchskontexten von Himmelmann (1997) werden auch die Strategien des Chinesischen kurz erläutert, um die definite Interpretation der Nominalphrasen zu bewirken. Im Abschnitt 4 werden einige Studien und deren Ergebnisse zum Erwerb des Artikelsystems durch Lernende mit artikellosen L1-Sprachen referiert. Die eigene empirische Studie über den Gebrauch des Definitartikels von chinesischen L2-Lernenden des Deutschen wird im Abschnitt 5 dargestellt. Ein abschließender Teil fasst die Ergebnisse zusammen.

\section{Lernen im L2-Erwerb: positiver und negativer Transfer}

In ihrer wichtigen Studie stellen Klein \& Perdue (1992, S. 313; 1997) fest, dass der Erwerbsprozess in der L2 genauso wie der Mutterspracherwerb durch universelle Tendenzen - unabhängig von der gesprochenen L1 - geprägt ist. In der ersten Phase des L2-Erwerbs, die die Autoren Basic Variety nennen, hat die L1 noch keinen Einfluss. In der Phase der Basic Variety wird vor allem das lexikalische Material gelernt und das sprachliche Material wird organisiert, aber die Flexion ist noch nicht erworben. Nach Watorek, Benazzo \& Hickmann (2012, S. 3) sind Kohärenzstrategien in dieser Phase noch sehr elementar, z.B. wird die Null-Anapher oft fälschlicherweise eingesetzt, was zu Ambiguitäten führt. Erst bei fortgeschrittenen Lernenden werden syntaktische Strategien, die der Kohärenz dienen, korrekt verwendet, wie etwa der zielsprachliche Gebrauch von Anaphern oder von Konnektoren. Allerdings intervenieren bei einer höheren Kompetenz auch die Muster der L1.

Anders ist es beim Lernen des Artikelsystems. Da scheint die L1 bereits in der Basic Variety den Gebrauch des Artikels zu beeinflussen. Nach Master (2002, S. 332), der sich mit dem Erwerb des Artikelsystems im Englischen als Fremdsprache beschäftigt, sind vor allem drei Ursachen für die Schwierigkeiten im Artikelerwerb verantwortlich. Zum einen gehören die Artikel (ein, der) zu den am häufigsten vorkommenden Funktionswörtern in der Sprache, die die kontinuierliche bewusste Anwendung von Regeln über einen längeren Diskurs hinweg erschweren. Ferner sind Funktionswörter normalerweise nicht betont und daher für einen Lernenden sehr schwer in der gesprochenen Sprache zu unterscheiden. Schließlich werden im Artikelsystem mehrere Funktionen auf ein einzelnes Morphem geladen, was eine erhebliche Belastung für den Lernenden darstellt, der im Allgemeinen nach einer 1-zu-1 Entsprechung zwischen Form und Funktion sucht. 
Nach Filipović \& Hawkins (2013, S. 10) spielen beim Lernen in der L2 sehr unterschiedliche und komplexe Faktoren eine Rolle, die das Lernen erleichtern oder erschweren. Sie schlagen deshalb ein multifaktorielles Modell der L2-Erlernbarkeit vor, das sie Complex Adaptive System Principle (CASP) nennen, in dem Faktoren wie Transfer, Komplexität und Frequenz der grammatischen Strukturen sowie Verarbeitungsmechanismen berücksichtigt werden. Das Lernen im L2-Erwerb wird nach Filipović \& Hawkins (2013, S. 10) durch verschiedene Prinzipien geleitet. Eines davon und zugleich auch das wichtigste, allgemeinste Prinzip ist das Minimize Learning Efforts, das besagt, dass "learners of a second language (L2) prefer to minimize learning effort when they learn the grammatical and lexical properties of the L2." Nach diesem Prinzip wird der Lernaufwand minimiert, wenn beispielsweise lexikalische oder grammatische Eigenschaften der L1 auf die L2 übertragen werden - im Falle, dass diese gleich sind. Ein weiteres Beispiel ist das Filtern von negativem Transfer aus der L1, wenn die transferierten Eigenschaften für die Kommunikation in der L2 hinderlich sind. Mehrere Studien zum positiven und negativen Transfer scheinen das Prinzip Minimize Learning Effort zu bestätigen. Gerade im Bereich Artikelerwerb zeigt die Untersuchung von Sleeman (2004), dass das Artikelsystem des Französischen mit größerer Schnelligkeit bzw. geringerer Fehlerhäufigkeit von niederländischen L2-Lernern erworben wird, die ein Artikelsystem in ihrer L1 haben, als von japanischen L2-Lernern, die keine Definitartikel aus ihrer L1 kennen. In mehreren Studien wird immer wieder bestätigt, dass gerade das Merkmal Definitheit bzw. Spezifizität auch bei fortgeschrittenen Lernern schwierig zu erlernen ist, wenn diese Eigenschaften in der eigenen L1 nicht morphosyntaktisch realisiert werden. Dies zeigen auch die Ergebnisse der empirischen Untersuchung in Filipović \& Hawkins (2013) (auch schon in Hawkins \& Buttery 2010 sowie Hawkins \& Filipović, 2012), die in ihren Studien zum Erwerb des Definit- und Indefinitartikels des Englischen Lernende mit unterschiedlichen L1 in den verschiedenen Phasen des Lernprozesses getestet haben. Unter der L1 der untersuchten Personen sind solche mit einem Artikelsystem wie Französisch, Deutsch und Spanisch und solche wie Türkisch, Japanisch, Koreanisch und Russisch, die kein Artikelsystem haben. Während die Fehlerfrequenz bei der Realisierung des Artikels durch die erste Gruppe in allen Erwerbsphasen relativ niedrig ist, ist die nichtzielsprachliche Realisierung des Artikels bei der zweiten Gruppe signifikant höher. Obwohl die Korrelation zwischen artikelloser L1 und Fehlerfrequenz relativ deutlich ist, bedeutet dies nach Filipović \& Hawkins (2013) nicht unbedingt, dass auch eine Korrelation zwischen der Präsenz eines Artikelsystems in der L1 und der niedrigen Fehlerfrequenz besteht. Neben fehlenden Artikeln kann in vielen Fällen auch ein Übergebrauch des Indefinitartikels durch diese Lernenden (Leung, 2001, lonin, Ko \& Wexler, 2004) oder die 
Verwendung des Definitartikels anstelle des Indefinitartikels (Master, 1987; Young, 1996) festgestellt werden. Sprecher des Mandarin verwenden auch andere Elemente wie Demonstrativa oder Numeralia an der Stelle des englischen Artikels (Robertson, 2000). In all diesen Fällen wirkt sich die L1 durch negativen Transfer auf den Erwerb von L2Eigenschaften aus. Aber worin genau unterscheiden sich artikellose Sprachen wie das Chinesische von Sprachen, die ein Artikelsystem aufweisen? Wie wird in artikellosen Sprachen die definite Interpretation einer Nominalphrase bewirkt?

\section{Definite Interpretation der Nominalphrasen in artikelhaltigen und artikellosen Sprachen}

In vielen Grammatiken werden Definitartikel als die morphosyntaktische Realisierung des Merkmals Definitheit bzw. Spezifizität definiert. Dabei existieren viele Sprachen in der Welt, die keinen Definitartikel oder gar kein Artikelsystem aufweisen, wie z.B. das Chinesische oder das Persische. Während im Deutschen der Definitartikel die Definitheit der Nominalphrasen markiert (1a.), werden definite Nominalphrase im Chinesischen (1b.) sowie auch im Persischen (1c.) als sogenannte,nackte' Nomen realisiert:

(1) a. Der Hund rannte weg. / *Hund rannte weg. (Deutsch)

b. Gǒu pǎo-zǒu-le.

(Chinesisch)

Hund

renn-geh-PERF

c. Sag

farar kard.

(Persisch)

Hund

rannte weg

Eine Vielzahl von Sprachen, die über kein Artikelsystem verfügen, realisiert Definitheit durch Wortstellungsregularitäten oder Kasus wie das Russische oder durch die Kongruenz zwischen Verb und Objekt wie beispielsweise Swahili. Das Chinesische benutzt in einigen Fällen auch Demonstrativpronomina.

Kramsky (1972) hat eine Typologie von sieben Sprachtypen vorgeschlagen, in der die Kategorien ,Determination' und ,Indetermination' anhand der verschiedenen formalen Mittel klassifiziert werden. Unter Typ A fallen die Sprachen, deren Determination und Indetermination durch freie Morpheme realisiert werden, dazu gehören z. B. die deutschen bestimmten Artikel der, die, das und die unbestimmten Artikel ein, eine, ein. Vom Typ B sind Sprachen, die entweder freie Morpheme oder proklitische und enklitische Elemente als Markierung verwenden; z. B. sind im Dänischen die enklitischen Formen -en und -et 
Definitheitsmarkierungen, Indetermination jedoch wird durch indefinite Artikel ausgedrückt. Sprachen vom Typ C haben nur proklitische und enklitische Elemente. Determination und Indetermination im Sprachtyp D sind im Nomen selbst inhärent. In Typ E wird die Kategorie der Definiheit/Indefinitheit an der Flexion realisiert. Phonologische Mittel wie Betonung und Intonation spielen eine Rolle in Sprachtyp F. Zum Sprachtyp G gehört das Chinesische, in dem die Kategorien Determination und Indetermination keine morphosyntaktische Realisierung kennen. Es stellt sich dabei die Frage, wie Lernende des Deutschen, die eine Muttersprache vom Typ G haben, das Artikelsystem in der L2 erwerben. Auf diese Frage wird in Abschnitt 5 eingegangen, in dem die empirische Studie mit Daten von chinesischen Lernern der L2 Deutsch diskutiert wird.

Definitheit ist ein komplexer Begriff, der eine weitere und eine engere Definition kennt. Im weiteren Sinn werden Nominalphrasen als definit verstanden, deren Referenten identifizierbar sind. Dazu zählen Personalpronomina, Eigennamen, Demonstrativa, Universalquantoren, Possessiva sowie Nominalphrasen mit Definitartikel (Lyons, 1999, S. 278). Da fast alle Sprachen über eines der oben genannten Sprachmittel verfügen, wird Definitheit im weiteren Sinn als sprachuniversal betrachtet. Im engeren Sinn bedeutet Definitheit die Grammatikalisierung der Identifizierbarkeit. Nur wenn Definitheit in einer Sprache durch typische grammatische Morpheme wie Affixe, Klitika oder morphophonologisch schwache Formen markiert ist, z. B. durch die Definitartikel im Deutschen, kann davon ausgegangen werden, dass diese Kategorie in einer Sprache morphosyntaktisch existiert. Von diesem Standpunkt aus betrachtet ist Definitheit ein Spezifikum mancher Sprachen, denn nicht alle Sprachen verfügen über die grammatischen Sprachmittel, die zur Markierung der Definitheit dienen. Typologisch betrachtet weisen die wenigsten Sprachen der Welt ein Artikelsystem auf.

\subsection{Definitheit im Deutschen}

Definitheit ist ein weitaus komplexerer Begriff, als man ihn nur durch die Realisierung des Definitartikels definieren kann, denn zur Definitheit gehören Eigenschaften wie u.a. Identifizierbarkeit, Familiarität, Einzigkeit. Nach Lyons können allerdings alle Merkmale auf Identifizierbarkeit reduziert werden. Wenn der Referent einer Nominalphrase identifiziert werden soll, müssen genügend Informationen vorliegen, damit der Referent von anderen unterschieden werden kann. Diese Informationen bestehen hauptsächlich aus zwei Kategorien. Die eine ist 'direct physical or linguistic co-presence', die andere 'shared background knowledge' (Chen, 2004, S. 1136): 
(2) Wer ist diese Frau?

(3) Er hat ein gebrauchtes Auto gekauft. Das Horn funktioniert nicht.

Bei der 'linguistic co-presence' wie im Beispiel (1) oben muss der Referent,Hund' vom Sprecher schon im Kontext eingeführt worden sein. Nachdem ein Referent im Kontext eingeführt wird, kann dieser als identifizierbar betrachtet werden. Im Fall 'direct physical co-presence' befindet sich der Referent in einer Situation, in der sowohl Sprecher als auch Hörer anwesend sind. Der Referent kann mit oder ohne begleitende paralinguistische Ausdrücke wie Mimik und Gestik identifiziert werden. Im Beispiel (2) sieht die Situation vielleicht so aus: Der Sprecher und der Hörer sind in einem Zimmer und eine Frau kommt herein. Der Sprecher kann den Hörer mit Gestik oder Mimik fragen, wer die hereingekommene Frau ist. Zudem spielt 'shared background knowledge' auch eine Rolle bei dem Identifizieren eines Referenten. Im Beispiel (3) nimmt der Sprecher Bezug auf das Wissen, dass ein Auto ein Horn hat. In dieser Situation kann das Horn vom Hörer ohne Schwierigkeit identifiziert werden. Solche Informationen gehören zum allgemeinen Weltwissen, welches entweder episodisch, spezifisch oder generisch ist (Chen, 2004, S. 1136-1139).

Die weitere Eigenschaft der Familiarität kann auch der Identifizierbarkeit zugeordnet werden. Familiarität gilt, wenn der Referent nicht nur dem Sprecher, sondern auch dem Hörer bekannt ist. Aber "definiteness of the noun phrase confirms an association which is only probable or possible rather than known" (Lyons, 1999, S. 4). Die Eigenschaft der Familiarität ist nicht ausreichend für die Erklärung der Verwendung des bestimmten Artikels. Es gibt Nominalphrasen, deren Referenten allein dem Sprecher bekannt sind, wie im folgenden Satz belegt wird:

(4) The president of Ghana is visiting tomorrow. (Lyons, 1999, S. 3)

Aus dem allgemeinen Wissen weiß der Hörer vielleicht, dass es einen Präsidenten in Ghana gibt. Das bedeutet jedoch nicht, dass er den Präsidenten kennt.

Eine andere wichtige Eigenschaft ist die Einzigkeit. Mit dem Begriff Einzigkeit wird der Tatsache Rechnung getragen, dass der bestimmte Artikel den einzigen Referenten im Kontext signalisieren kann. 
Die gerade diskutierten Eigenschaften können als mehr oder weniger universal betrachtet werden. Allerdings bestehen spezifische Verwendungsweisen des Definitartikels auch in Sprachen, die zum Typ A gehören. Gerade generische Nomina weisen eine breite Variation auf (Barton, Kolb \& Kupisch, 2015). Im Deutschen werden generische Nomina im Singular - wie im Englischen - durch den Definitartikel markiert (5a.), der Indefinitartikel ist hingegen nicht akzeptabel (5b.) ${ }^{4}$ :

(5) a. Der Löwe ist ein gefährliches Tier.

b. ??Ein Löwe ist ein gefährliches Tier.

c. Die Löwen sind gefährliche Tiere.

d. Löwen sind gefährliche Tiere.

Gerade mit generischen Nomen haben Lernende mit einer artikellosen L1 große Schwierigkeiten.

Wie Chen diskutiert auch Himmelmann (1997) den pragmatischen Aspekt der Identifizierbarkeit eines Referenten, denn die Interpretierbarkeit von Definitheit ist von mehreren Faktoren abhängig. Deshalb versucht Himmelmann (1997) eine Klassifikation der Gebrauchskontexte für den Definitartikel, die er dann in ,semantisch' und ,pragmatisch' unterteilt.

Himmelmann (1997, S. 39) unterscheidet folgende Gebrauchskontexte:

A. unmittelbar-situativer Gebrauch: Der Referent ist in der unmittelbaren Äußerungssituation erschließbar und sogar sichtbar wie im Beispiel (6):

(6) Reich mir bitte die Butter!

B. anaphorischer Gebrauch: Der Referent ist im Vortext erwähnt wie in (7):

(7) A: Hast du Butter gekauft?

4 Das Englische hingegen, das auch eine germanische Sprache ist, kann generische Nomina durch Definit(a.) und Indefinitartikel (b.) im Singular markieren oder aber das Nomen im Plural ,nackt' lassen, i.e. ohne jegliche Markierung (d.). Der Definitartikel im Plural ist hingegen nicht grammatisch (c.):

a. The lion is a dangerous animal.

b. A lion is a dangerous animal.

c. *The lions are dangerous animals.

d. Lions are dangerous animals. 
B: Ja, die Butter ist im Kühlschrank.

C. assoziativ-anaphorischer Gebrauch: Der Referent kann durch Assoziation zu einem zuvor erwähnten Referenten erschlossen werden, wie in metonymischen Verhältnissen vom Typ Haus - Dach/Tür/ Fenster:

(8) Ich habe ein Haus gekauft. Das Dach ist grün.

D. abstrakt-situativer Gebrauch: Der Referent kann aufgrund von Weltwissen erschlossen werden, wie z.B.:

die Sonne, der Mond, der Bundespräsident, die Zahl 3; (hier wird auch Einzigartigkeit markiert)

aber auch durch Superlative (der Schönste) oder durch Modifikatoren wie der Einzige, derErste;

E. generischer Gebrauch wie in (9):

(9) Der Hund ist der beste Freund des Menschen.

Die Gebrauchskontexte für den Definitartikel werden in den Beispielen A bis E immer abstrakter: Während im unmittelbar-situativen Kontext (A) der Referent in der Äußerungssituation direkt sichtbar und im anaphorischen Gebrauch zuvor erwähnt worden ist, muss der Hörer im assoziativ-anaphorischen Gebrauch (C) eine kognitive Leistung erbringen. Vor allem muss hierbei ein gemeinsames Wissen zwischen Hörer und Sprecher bestehen, damit der Referent eindeutig identifiziert werden kann. Ähnliches gilt für (D): Hier ist der Bezug zum Referenten noch etwas abstrakter, da der Referent nicht konkret, greifbar' ist. Schließlich wird in (E) mit dem generischen Gebrauch nicht auf einen konkreten Referenten Bezug genommen, sondern auf eine Gattung oder einen Typ. Das Substantiv der Hund im Singular steht für die ganze Klasse. Die Rede ist also nicht von einem konkreten Hund. Demzufolge unterscheidet Himmelmann zwischen semantischer und pragmatischer Definitheit. Die Gebrauchskontexte A und B werden von Himmelmann als ,pragmatisch', die Gebrauchskontexte C, D und E hingegen als ,semantisch' bezeichnet. 


\subsection{Definite Interpretation im Chinesischen}

Um zu verstehen, wie sich die L1 Chinesisch auf den Artikelerwerb im Deutschen auswirkt, soll hier ein knapper Überblick über die Kontexte gegeben werden, in denen Nomen im Chinesischen definit interpretiert werden. Chinesische Nomen werden meistens ,nackt' realisiert, das heißt, es bedarf keines Elements, um sie als definit oder indefinit zu markieren:

(10) Wǒ măi-le shū le.

Ich kaufe-PERF Buch PRT

'Ich habe ein Buch/einige Bücher/Bücher/die Bücher gekauft.'

Da das Chinesische eine isolierende Sprache ist, kann das nackte Nomen shū im Beispiel (10) definit sowohl im Singular als auch im Plural wie auch indefinit interpretiert werden. In (10) befindet sich das Nomen shū an postverbaler Stelle. In präverbaler Position (11a) allerdings wird das Nomen - unabhängig davon, ob in Subjekt- oder Objektfunktion - definit interpretiert: Es handelt sich um das Topik des Satzes:

(11) a. Ren lai le. SUBJEKT

Person komm-PERF

'Die Person ist gekommen.'
b. Lai le ren le.
Komm PERF Person PRT

'Einige Personen sind gekommen/Personen sind gekommen.'
(12) yifu wo yijing xiwan le. OBJEKT (aus Guan 2019: 34)
Wäsche ich schon waschen PERF
'Ich habe schon die Wäsche gewaschen'

Das Chinesische hat zwei Demonstrativpronomina, zhe 'dieser' und na 'jener', die in anaphorisch-situativen Kontexten das Nomen als definit markieren, wie in den folgenden Beispielen (aus Guan, 2019, S. 44):
(13) ni renshi Zhangsan ma?
zhe ge ren
hen conming
du kennen Zhangsan Frage-PRTCL diese $\mathrm{KL}$ Mensch sehr intelligent. 'Kennst du Zhangsan? Er ist sehr intelligent.' 
(14) wo mai le yi ben xiaoshuo. na ben xiaoshuo hen youqu. ich kaufen PRF ein KL Roman jene $\mathrm{KL}$ Roman sehr interessant. 'Ich habe einen Roman gekauft. Dieser Roman ist sehr interessant.'

Zudem weisen auch artikellose Sprachen wie artikelhaltige Sprachen Definitheitseffekte auf (Huang 1987, 2009), z.B. in existentiellen Konstruktionen wie in you-Konstruktionen:

(15) a. Youyi-ben shu zai zhuo-shang. (aus Yang, 2008, S. 120) haben ein-CL Buch auf Tisch-oben 'Es gibt ein Buch auf dem Tisch'

b. *You nei-ben shu zai zhuo-shang. haben dieses-CL Buch auf Tisch-oben

* 'Es gibt dieses Buch auf dem Tisch.'

Die Daten deuten also darauf hin, dass - auch wenn im Chinesischen Definitheit nicht morphosyntaktisch realisiert wird - es durchaus die Kategorie Definitheit im weiteren Sinne kennt. Im ersten Abschnitt wurde Definitheit in diesem Sinne als universelle Kategorie bezeichnet.

Interessanterweise gibt es Untersuchungen zu manchen chinesischen Dialekten, die zeigen, dass diese bereits eine Art Artikel aus dem Definitpronomen entwickeln (Guan 2019).

\section{Gebrauch der Definitartikel bei L2-Lernenden des Deutschen}

In ihrem Aufsatz gehen Schaeffer \& Matthewson (2005) davon aus, dass Sprachen, die mindestens einen Artikeltyp aufweisen, in zwei Gruppen unterteilt werden können: solche, die den Artikel aufgrund der Opposition definit-indefinit wählen, und solche, in denen die Opposition zwischen spezifisch-unspezifisch relevant ist. Zur ersten Gruppe gehören Sprachen wie Englisch, Deutsch oder Französisch. Nach Spezifizität organisiert ist z.B. die polynesische Sprache Samoanisch (Ionın \& Wexler, 2004). Lernende einer Fremdsprache müssen demzufolge zunächst diese Unterscheidung lernen.

Spezifizität ist vor allem beim Gebrauch des Indefinitartikels von Relevanz: Ein Nomen wird als spezifisch interpretiert, wenn der Referent dem Sprecher bekannt ist bzw. durch den Sprecher identifizierbar ist. Im Gegensatz dazu führt ein Nomen mit nicht-spezifischer 
Referenz ein durch den Sprecher nicht identifizierbares oder für den Sprecher nicht bekanntes Individuum in den Diskurs ein. Diese Art der Spezifizität kann durch die Kompatibilität mit Fortsetzungen des Satzes getestet werden, die die Identifizierbarkeit des Referenten durch den Sprecher explizieren (Geist, 2010):

(16) Ein Student hat in der Klausur geschummelt.

a. Ich kenne ihn, er heißt David. [identifizierbar durch den Sprecher = spezifisch]

b. Ich kenne ihn nicht und werde versuchen herauszufinden, wer das war. [nichtidentifizierbar $=$ nicht-spezifisch]

Die Fortsetzung in (a.) deutet darauf hin, dass der Referent von ein Student durch den Sprecher identifizierbar ist, während nach (b.) der Sprecher den Referenten nicht identifizieren kann.

Zahlreiche Studien (wie Ionin, Ko \& Wexler, 2004; Master, 1987; Parrish, 1987; Thomas, 1989; Murphy, 1997; Robertson, 2000; Schaeffer \& Matthewson, 2005 u.a.), die sich mit dem Erwerb von Definitheit bei L2-Lernenden des Englischen befassen, legen die semantische Unterscheidung zwischen definit/indefinit bzw. spezifisch/unspezifisch zugrunde. Die meisten beschäftigen sich mit der Typologie von Fehlern im Artikelgebrauch. Auch der Aufsatz von Breckle \& Zinsmeister (2010) untersucht die Frage, ob die Opposition definit/indefinit bzw. spezifisch/unspezifisch eine Rolle in der Produktion von deutschen Nominalphrasen durch chinesische Lernende des Deutschen spielt. Breckle \& Zinsmeister (2010) kommen zu folgendem Ergebnis: Während Definitheit ein wichtiger Faktor ist, spielt Spezifizität beim zielsprachlichen bzw. nichtzielsprachlichen Gebrauch des Artikels durch chinesische L2-Lernende des Deutschen kaum eine Rolle.

In meiner empirischen Studie über den Gebrauch des Definitartikels bei chinesischen L2-Lernenden des Deutschen habe ich mich auf die Interferenzstrukturen insbesondere im Bereich der Definitheit konzentriert.

\section{Gebrauch des Definitartikels bei chinesischen L2-Lernenden des Deutschen: eine empirische Studie}

Im Wintersemester 2013/14 waren ca. 30.500, im Wintersemester 2017/18 39.541 chinesische Studierende an deutschen Hochschulen eingeschrieben (Quelle: Statistisches 
Bundesamt). Die Chinesen stellen die größte Gruppe internationaler Studierender an deutschen Hochschulen dar, und auch die Nachfrage nach deutschen Kursen in China wächst. Der Grund für die hohe Zahl chinesischer Studierender in Deutschland und für das steigende Interesse an der deutschen Sprache ist, dass ein deutscher Abschluss sowie auch gute Deutschkenntnisse auf dem chinesischen Arbeitsmarkt Vorteile bringen. So suchen zum Beispiel viele deutsche Unternehmen in China händeringend nach chinesischen Fachkräften (Quelle: Deutschlandfunk).

Die didaktischen Materialien für den Unterrich der deutschen Sprache werden in China erstellt. Im Bacherlorstudiengang Germanistik wird zum Beispiel gerne das Buch Studienweg Deutsch vom Autor Liangmin (Foreign Language Teaching and Research Press) verwendet.

Die leitende Frage in meiner empirischen Untersuchung war: Inwiefern wirkt sich die L1 Chinesisch auf die L2 Deutsch beim Gebrauch des Definitartikels aus?

Das Korpus bestand aus den Motivationsschreiben von 80 chinesischen Bewerberinnen für den Masterstudiengang Germanistik: Sprachwissenschaft an der Otto-Friedrich-Universität Bamberg im Zeitraum zwischen dem Wintersemester 2014/15 und dem Sommersemester 2018.

Alle Bewerberinnen im Alter von 23-25 Jahren hatten ein 4-jähriges Bachelorstudium der Germanistik an einer chinesischen Universität erfolgreich absolviert und mussten TestDaF 4x4 bestanden haben, um sich an der Universität Bamberg bewerben zu können. Es handelte sich also entsprechend dem absolvierten Test um fortgeschrittene Lernerinnen. Sowohl während des Studiums als auch nach dem Studium der Germanistik haben jedoch selbst fortgeschrittene chinesische Lerner des Deutschen Schwierigkeiten mit dem zielsprachlichen Gebrauch des Definitartikels. Ein Beispiel aus einem der untersuchten Motivationsschreiben wird in (17) gegeben: Dort werden die Nominalphrase ,Literaturrecherche', ,Universität Bamberg' und ,Naturwissenschaft' sowie ,philosophische und sprachliche Wissenschaft' nicht-zielsprachlich artikellos realisiert:

(17) In Literaturrecherche habe ich eine Masterarbeit, die von einem Studenten der Universität Bamberg angefasst, zitiert. Danach habe ich mich über Universität Bamberg sehr genau informiert. Soweit ich weiß, Universität Bamberg ist eine 
Universität mit langer Tradition und bietet vielfältige Studienangebote an, die von Naturwissenschaft bis philosophische und sprachliche Wissenschaft reichen. (GE01_07)

Die leitenden Fragen bei der Analyse der Daten sind die folgenden gewesen:

1. In welcher Frequenz treten ziel- bzw. nicht-zielsprachliche Realisierungen des Definitartikels bei fortgeschrittenen chinesischen Lernerinnen des Deutschen auf?

2. Unter welche Kategorie nach Himmelmann (1997) fallen solche ziel- und nichtzielsprachliche Realisierungen?

3. Sind die nicht-zielsprachlichen Realisierungen auf negativen Transfer zurückzuführen?

4. Welche Kategorie scheint besonders große Schwierigkeiten zu bereiten?

Aus dem zusammengestellten Korpus wurden alle enthaltenen Nominalphrasen gesammelt und klassifiziert. Die im Korpus enthaltenen Nominalphrasen sind nach den Kategorien zielsprachlich vs. nicht-zielsprachlich, darunter Nominalphrasen mit Definitartikel, mit Indefinitartikel und artikellos klassifiziert. Insgesamt sind in den untersuchten Texten 497 Nominalphrasen enthalten: 281 mit Definit-, 111 mit Indefinitartikel und 105 artikellos. In der folgenden Tabelle sind die jeweiligen zielsprachlichen und nicht-zielsprachlichen Realisierungen auch prozentual angegeben:

Tabelle 1: Realisierung des Artikels bei chinesischen Studierenden

\begin{tabular}{|l|l|l|}
\hline & zielsprachlich & nicht-zielsprachlich \\
\hline Definitartikel & $233(82,9 \%)$ & $48(17,1 \%)$ \\
\hline Indefinitartikel & $86(77,5 \%)$ & $25(24,5 \%)$ \\
\hline artikellos & $78(74,3 \%)$ & $27(25,7 \%)$ \\
\hline
\end{tabular}

Bezüglich der ersten Fragestellung scheinen die Daten die von Hawkins \& Buttery (2010) sowie von Hawkins \& Filipović (2012) festgestellte Fehlerfrequenz bei der Realisierung der Indefinitartikel dahingehend zu bestätigen, dass die chinesischen Studentinnen vor allem mit indefiniten Nominalphrasen die größten Schwierigkeiten haben. Eine Überrealisierung des Indefinitartikels konnte jedoch nicht festgestellt werden. Da jedoch der Schwerpunkt dieses Beitrags auf den definiten Nominalphrasen liegt, werde ich den Gebrauch des Indefinitartikels nicht weiter vertiefen.

In einem weiteren Schritt sind sowohl die zielsprachlichen als auch die nichtzielsprachlichen realisierten NPs nach den von Himmelmann (1997) vorgeschlagenen 
Klassen unterteilt, um - wie in der zweiten Fragestellung zur Diskussion gestellt herauszufinden, mit welcher Klasse/mit welchen Typen der Gebrauchskontexte die chinesischen Lernerinnen die größten Schwierigkeiten haben. Die Daten sind noch nicht sehr belastbar, aber es zeigen sich dabei deutliche Tendenzen.

Die Gebrauchskontexte, in denen der Definitartikel zielsprachlich realisiert wird, sind prozentual folgende:

- 60\% anaphorisch-kontextuell

- 10\% in einer Topikposition

- 15\% abstrakt-situativ

- $15 \%$ generisch (einzigartig)

Die Zahlen entsprechen der Frequenz bei der zielsprachlichen Realisierung des Definitartikels in den einzelnen Gebrauchskontexten.

Die dritte Frage bezüglich des negativen Transfers aus der L1 scheint interessante Ergebnisse zu liefern. Der anaphorisch-kontextuelle Gebrauch ist am wenigsten von Fehlern betroffen. Bei diesem Kontext scheinen die chinesischen Lernenden keine großen Schwierigkeiten zu haben, den Definitartikel zielsprachlich zu verwenden. Im Chinesischen wird gerade in diesem Kontext das Demonstrativum na ,dieser' als morphosyntaktisches Mittel verwendet, um das Nomen als definit zu markieren.

Der abstrakt-situative Kontext ist für chinesische Lernende besonders schwierig. Zum Beispiel handelt es sich im folgenden Satz aus einem der Korpustexte mit,Rathaus' um einen öffentlichen Ort, den es in der Stadt nur einmal gibt. Aus diesem Grund wäre der Indefinitartikel nicht geeignet. So entscheidet sich der Lernende für den NullArtikel:

(18) Morgen hole ich meine neue Aufenthaltstitel in Rathaus. (GE03_05)

Tendenziell realisieren also selbst fortgeschrittene chinesische Lerner Nomen als ,nackt', wenn diese zunächst schon erwähnt worden sind und deren Interpretation kontextuell zu rekonstruieren ist. Ähnliches gilt für die Topikposition. Die pragmatischen Gebrauchskontexte sind demzufolge die häufigsten unter den zielsprachlichen Realisierungen des Definitartikels. Unter den semantischen Kontexten, die hingegen 
noch Schwierigkeiten bereiten, finden sich häufig auch Nomina, die eine generische Interpretation haben.

Ich habe - bislang nur stichprobenartig - einige Daten in den späteren Texten der gleichen Studierenden untersucht, um die Entwicklung im Lernprozess der Studierenden genauer zu betrachten. Dabei konnte festgestellt werden, dass auch bei der individuellen Entwicklung im Erwerb der Definitheitsmarkierung generische Nomina relativ lange und persistent ohne Artikel realisiert werden. Eine ausdifferenziertere Betrachtung wäre hier notwendig.

So zeigt sich, dass auch im L2-Erwerb Lernerphasen festzustellen sind. Entsprechend sollten auch die Materialien so konzipiert werden, dass die einzelnen Kategorien der Gebrauchskontexte bewusst gemacht und geübt werden.

\section{Schluss}

In diesem Beitrag ist die Realisierung der Definitheit bei fortgeschrittenen chinesischen Lernenden des Deutschen in einer empirischen Pilotstudie untersucht worden. Definitheit ist ein sehr komplexer Begriff, der im weiteren Sinne als universelle Kategorie in den Sprachen der Welt betrachtet werden kann. Im engeren Sinne jedoch unterscheiden sich Sprachen erheblich in der grammatischen Realisierung dieser Kategorie. So weisen Sprachen wie das Deutsche oder das Englische ein Artikelsystem für die Merkmale [definit] und [indefinit] auf, während andere Sprachen wie das Chinesische keinen Definitartikel kennen, dafür aber andere sprachlichen Mittel verwenden, um die definite Interpretation eines Nomens zu bewirken. Diese unterschiedliche grammatische Umsetzung der Kategorie Definitheit stellt für L2-Lerner des Deutschen, die eine artikellose L1 sprechen wie die chinesischen Lerner, eine große Schwierigkeit dar. Denn selbst fortgeschrittene Lerner produzieren noch relativ häufig nicht-zielsprachliche Nominalphrasen mit der Auslassung des Definitartikels. Die empirische Studie hat gezeigt, dass vor allem in den von Himmelmann (1997) als pragmatisch bezeichneten Gebrauchskontexten der Definitartikel am häufigsten ausgelassen wird. Auch die generischen Nomina bleiben relativ lange ,nackt' realisiert. Wichtig wäre es also, dass auch die DaF-Materialien zum Gebrauch des Definitartikels auf die verschiedenen Gebrauchskontexte Bezug nehmen und entsprechende Übungen anbieten.

Finanzielle Förderung: Dieser Beitrag wurde von keiner Institution finanziell unterstützt. 


\section{Literaturverzeichnis}

Barton, D., Kolb, N. \& Kupisch T. (2015). Definite article use with generic reference In German: an empirical study.

Zeitschrift für Sprachwissenschaft 34.2, 147-173.

Breckle, M. \& Zinsmeister, H. (2010). Zur lernersprachlichen Generierung referierender Ausdrücke in argumentativen

Texten. Skiba D. (Hrsg.) Textmuster: schulisch-universitär-kulturkontrastiv. Frankfurt/Main: Peter Lang. 79-101.

Chen, P. (2004). Identifiability and definiteness in Chinese. Linguistics 42.6, 1129-1184.

Filipović, L. \& Hawkins, J. A. (2013). Multiple factors in second language acquisition: the CASP model.

Geist, L (2010). Indefinitpronomina im Russischen und Spezifizität. Zeitschrift für Slawistik 55.2, 206-222.

Guan, J. (2019) Kontrastive Untersuchung zur Realisierung der Definitheit im Deutschen und im Chinesischen.

Dissertation Universität Mainz.

Hawkins, J. A., \& Buttery, P. (2010). Criterial features in learner corpora: Theory and illustrations. English Profile Journal, 1.

Hawkins, J. A., \& Filipović, L. (2012). Criterial Features in L2 English: Specifying the Reference Levels of the Common European Framework (Vol. 37). Amsterdam: John Benjamins.

Himmelmann, N. (1997). Deiktikon, Artikel, Nominalphrase. Zur Emergenz syntaktischer Struktur. Tübingen: Niemeyer.

Huang, C.-T. (1987). Existential sentences in Chinese and (in)definiteness. In E. J. Reuland / A. G. B. ter Meulen (eds.) The Representation of (In)definiteness. Cambridge, Mass.: The MIT Press. 226-253.

Huang, C.-T. et al. (2009). The syntax of Chinese. Cambridge: Cambridge University Press.

Ionin, T., Ko, H. \& Wexler, K. (2004). Article semantics in L2-acquisition: the role of specificity. Language Acquisition $12,3-69$

Ionin, T., Baek, S., Kim, E., Ko, H. \& Wexler, K. (2012). That's not so different from the: definite and demonstrative descriptions in second language acquisition. Second Language Research, 28, 69-101.

Ionin, T., Ko, H., \& Wexler, K. (2004). Article semantics in L2 acquisition: The role of specificity. Language Acquisition, 12(1), 3-69.

Klein, W., \& Perdue, C. (eds.) (1992). Utterance Structure: Developing Grammars Again. Amsterdam: John Benjamins.

Klein, W. \& Perdue, C. (1997). The Basic Variety (or: couldn't natural languages be much simpler?). Second Language Research, 13, 301-347.

Kramsky, J. (1972). The article and the concept of definiteness in language. Mouton: The Hague.

Leung, Y. I. (2001). The initial state of L3A: Full transfer and failed features? In X. Bonch-Bruevich, W. Crawford, J.

Hellerman, C. Higgins, / H. Nguyen (eds.) The Past, Present, and Future of Second Language Research: Selected Proceedings of the 2000 Second Language Research Forum. Somerville, M.A.: Cascadilla Press, 55-75.

Lyons, Ch. (1999). Definiteness. Cambridge: Cambridge University Press. 3-12, 278-279, 668.

Master, P. (1987). A Cross-Linguistic Interlanguage Analysis of the Acquisition of the English Article System, Doctoral dissertation, University of California, Los Angeles. 
Master, P. (2002). Information structure and English article pedagogy. System 30, 331-348.

Murphy, S. (1997). Knowledge and Production of English Articles by Advanced Second Language Learners, Doctoral dissertation, University of Texas, Austin.

Parrish, B. (1987). A New Look at Methodologies in the Study of Article Acquisition for Learners of ESL. Language Learning 37, 361-383.

Robertson, D. (2000). Variability in the Use of the English Article System by Chinese Learners of English. Second Language Research 16, 135-172.

Schaeffer, J. \& Matthewson, L. (2005). Grammar and pragmatics in the acquisition of article systems. Natural Language and Linguistic Theory 23.1, 53-101.

Sleeman, P. (2004). Guided learners of French and the acquisition of emphatic constructions. International Review of Applied Linguistics in Language Teaching, 42, 129-152.

Thomas, M. (1989). The Acquisition of English Articles by First- and Second-Language Learners. Applied Psycholinguistics 10, 335-355.

Watorek, M., Benazzo, S. \& Hickmann, M. (2012). New comparative perspectives in the study of language acquisition - Clive Perdue's legacy. In M. Watorek, S. Benazzo \& M. Hickmann (eds.) Comparative Perspectives on Language Acquisition: Tribute to Clive Perdue. Bristol: Multilingual Matters, 324-349.

Yang, N. (2008). The indefinite object in Mandarin Chinese: Its marking, interpretation and acquisition. Utrecht: LOT. Young, R. (1996). Form-function relations in articles in English interlanguage. In R. Bayley \& D. R. Preston (eds.) Second Language Acquisition and Linguistic Variation. Amsterdam: John Benjamins, 135-175. 